\title{
Identification of Virulence Factors Genes in Escherichia coli Isolates from Women with Urinary Tract Infection in Mexico
}

\author{
Daniela A. López-Banda, ${ }^{1}$ Erika M. Carrillo-Casas, ${ }^{2}$ \\ Margarita Leyva-Leyva, ${ }^{2}$ Gabriel Orozco-Hoyuela, ${ }^{3}$ Ángel H. Manjarrez-Hernández, ${ }^{4}$ \\ Sara Arroyo-Escalante, ${ }^{2}$ David Moncada-Barrón, ${ }^{5}$ Silvia Villanueva-Recillas, ${ }^{5}$ \\ Juan Xicohtencatl-Cortes, ${ }^{6}$ and Rigoberto Hernández-Castro ${ }^{1}$
}

${ }^{1}$ Department of Ecology of Pathogen Agents, Hospital General “Dr. Manuel Gea González”, 14080 Tlalpan, DF, Mexico

${ }^{2}$ Department of Molecular Biology and Histocompatibility, Hospital General "Dr. Manuel Gea González", 14080 Tlalpan, DF, Mexico

${ }^{3}$ Institute of Cell Physiology, Universidad Nacional Autónoma de México, 04510 Coyoacán, DF, Mexico

${ }^{4}$ Department of Public Health, Universidad Nacional Autónoma de México, 04510 Coyoacán, DF, Mexico

${ }^{5}$ Clinical Laboratory, Hospital General "Dr. Manuel Gea González", 14080 Tlalpan, DF, Mexico

${ }^{6}$ Department of Infectology, Hospital Infantil de México "Federico Gómez", 06720 Cuauhtémoc, DF, Mexico

Correspondence should be addressed to Rigoberto Hernández-Castro; rigo31@yahoo.com

Received 11 February 2014; Accepted 20 April 2014; Published 5 May 2014

Academic Editor: Angel Cataldi

Copyright (C) 2014 Daniela A. López-Banda et al. This is an open access article distributed under the Creative Commons Attribution License, which permits unrestricted use, distribution, and reproduction in any medium, provided the original work is properly cited.

E coli isolates (108) from Mexican women, clinically diagnosed with urinary tract infection, were screened to identify virulence genes, phylogenetic groups, and antibiotic resistance. Isolates were identified by MicroScan4 system; additionally, the minimum inhibitory concentration (MIC) was assessed. The phylogenetic groups and 16 virulence genes encoding adhesins, toxins, siderophores, lipopolysaccharide (LPS), and invasins were identified by PCR. Phylogenetic groups distribution was as follows: B1 9.3\%, A 30.6\%, B2 55.6\%, and D 4.6\%. Virulence genes prevalence was ecp 98.1\%, fimH 86.1\%, traT 77.8\%, sfa/focDE 74.1\%, papC $62 \%$, iutA 48.1\%, fyuA 44.4\%, focG 2.8\%, sfaS 1.9\%, hlyA 7.4\%, cnf-1 6.5\%, cdt-B 0.9\%, cvaC 2.8\%, ibeA 2.8\%, and rff $0.9 \%$. Regarding antimicrobial resistance it was above $50 \%$ to ampicillin/sulbactam, ampicillin, piperacillin, trimethoprim/sulfamethoxazole, ciprofloxacin, and levofloxacin. Uropathogenic E. coli clustered mainly in the pathogenic phylogenetic group B2. The isolates showed a high presence of siderophores and adhesion genes and a low presence of genes encoding toxins. The high frequency of $p a p C$ gene suggests that these isolates have the ability to colonize the kidneys. High resistance to drugs considered as first choice treatment such as trimethoprim/sulfamethoxazole and fluoroquinolones was consistently observed.

\section{Introduction}

Urinary tract infections (UTI) are one of the most common infections worldwide. Uropathogenic Escherichia coli (UPEC) is the primary pathogen causing UTIs; it colonizes the human intestine a few hours after birth and is considered part of the normal microbiota. However, it can cause various diseases such as diarrhea, UTI, and meningitis [1]. It is classified into three groups: (i) commensal, (ii) intestinal pathogenic, and (iii) extraintestinal pathogenic [2], and phylogenetically it has been classified into four classic groups
(A, B1, B2, and D) [3]. Uropathogenic E. coli is located within the extraintestinal pathogenic E. coli (ExPEC), classified primarily into the phylogenetic group B2 and to a lesser extent to group $\mathrm{D}$, whereas commensal strains are within the phylogenetic groups A and B1 [4-8].

The ability of $E$. coli to colonize different anatomical sites is due in part to genome plasticity and remodeling by acquisition or loss of genetic material from which it acquired resistance or virulence factors. Therefore, horizontal transfer is an important factor in the evolution and adaptation of E. coli to different niches $[9,10]$. The interaction between 
bacteria and epithelial cells is a multifactorial and complex phenomenon which involves several adhesins produced according to the stage of infection, while adherence to epithelial cells is essential for successful colonization and establishment; the expression of other genes encoding toxins, siderophores, lipopolysaccharide (LPS), capsule, and invasins determines the disease severity and the strain's virulence [8]. UPEC strains can cause acute infections and recurrent infections that do not respond to common antimicrobial treatments. UTI treatment generally includes $\beta$-lactam antibiotics, fluoroquinolones, or trimethoprim/sulfamethoxazole [11-13] but may vary according to patient age, sex, Pathogen involved, course of disease, and the urinary tract anatomic area involved [5]. The increased resistance may be related to changes in the bacterial genome by mutation or acquisition by horizontal transfer of an extrachromosomal or chromosomal material [14-16].

Urinary tract successful invasion depends on the bacteria virulence, inoculums size, and the host's defense mechanisms [18]. However, women have higher UTI's prevalence and incidence mainly due to their anatomical characteristics such as the proximity between the anus and the urethral opening, hormone effects, and changes in the genital microbiota [14, 19]. Clinically a UTI is defined by a bacteriuria with a count in midstream urine culture $\geq 10^{5} \mathrm{CFU} / \mathrm{mL}$ and pyuria or the presence of white blood cells in the urine, more than five leukocytes per field [19].

Globally it is estimated that about 150 million UTIs occur annually [20]. In the United States and Spain the current situation and treatment of urinary tract infections had been thoroughly described $[6-8,18]$; it is estimated that $11 \%$ of women experience at least one diagnosis of urinary tract infection (UTI) per year, and $60 \%$ of women will have or have had an UTI or more during their lifetime [4]. In Mexico UTI's status has not been described. However, to our knowledge it is E. coli one of the pathogenic agents of UTI and it is more frequent in women, with high incidence and prevalence, representing a costly problem for the health sector $[4,21]$. In 2008, 3,244,994 cases were reported, which represents an incidence of $3,041.7 / 100,000$ inhabitants, from which $75.6 \%$ $(2,453,608 / 100,000$ inhabitants $)$ were women, representing an incidence of 4,508.6/100,000.

The present study aimed to describe the profile of $E$. coli from Mexican women with urinary tract infection by the identification of virulence genes (fimH, papC, sfa/focDE, sfaS, focG, ecpA, ecpR-B, hlyA, cnf-1, cdt-B, cvaC, iutA, ibeA, $r f c$, $\operatorname{trat} T$, and $f y u A$ ), phylogenetic group, and their resistance to antibiotics to guide better diagnosis and treatment of UTI.

\section{Material and Method}

2.1. Bacteria and Culture. Bacterial isolates (108) were obtained from urine samples from women diagnosed with acute urinary tract infection and confirmed by the clinical laboratory of the General Hospital "Dr. Manuel Gea Gonzalez" during 2008 and until 2010. All samples with counts over $100,000 \mathrm{UFC} / \mathrm{mL}$ were included. Patients were within an age range between 12 and 58 years and mean age
TABLE 1: PCR primer for each virulence factor. Primer sequence was taken from Johnson and Stell, 2000 [5] and Blackburn et al., 2009 [17].

\begin{tabular}{|c|c|}
\hline Genes & Primer $\left(5^{\prime}-3^{\prime}\right)$ \\
\hline \multirow{2}{*}{$\operatorname{ecp} A$} & TGA AAA AAA AGG TTC TGG CAA TAG C \\
\hline & CGC TGA TGA GGA GAA AGT GAA \\
\hline \multirow{2}{*}{$\operatorname{ecpRB}$} & GTC ACA TGG CAA AAT GAT TAC AGC \\
\hline & TCA CGG GAA TGA ACT TAT CAC CC \\
\hline \multirow{2}{*}{ papC } & GTG GCA GTA TGA GTA ATG ACC GTT A \\
\hline & ATA TCC TTT CTG CAG GGA TGC AAT A \\
\hline \multirow{2}{*}{$s f a S$} & GTG GAT ACG ACG ATT ACT GTG \\
\hline & CCG CCA GCA TTC CCT GTA TTC \\
\hline \multirow{2}{*}{ focG } & CAG CAC AGG CAG TGG ATA CGA \\
\hline & GAA TGT CGC CTG CCC ATT GTC \\
\hline \multirow{2}{*}{$\mathrm{fimH}$} & TGC AGA ACG GAT AAG CCG TGG \\
\hline & GCA GTC ACC TGC CCT CCG GTA \\
\hline \multirow{2}{*}{$s f a / f o g D E$} & CTC CGG AGA ACT GGG TGC ATC TTA C \\
\hline & CGG AGG AGT AAT TAC AAA CCT GGC A \\
\hline \multirow{2}{*}{ cnfl 1} & AAG ATG GAG TTT CCT ATG CAG GAG \\
\hline & CAT TCA GAG TCC TGC CCT CAT TAT T \\
\hline \multirow{2}{*}{ hlyA } & AAC AAG GAT AAG CAC TGT TCT GGC T \\
\hline & ACC ATA TAA GCG GTC ATT CCC GTC A \\
\hline \multirow{2}{*}{$c d t-s$} & GAA AGT AAA TGG AAT ATA AAT GTC CG \\
\hline & GAA AAT AAA TGG AAC ACA CAT GTC CG \\
\hline \multirow{2}{*}{$c d t-a$} & AAA TCA CCA AGA ATC ATC CAG TTA \\
\hline & AAA TCT CCT GCA ATC ATC CAG TTT A \\
\hline \multirow{2}{*}{$\operatorname{colV}$} & CAC ACA CAA ACG GGA GCT GTT \\
\hline & CTT CCC GCA GCA TAG TTC CAT \\
\hline \multirow{2}{*}{ fyuA } & TGA TTA ACC CCG CGA CGG GAA \\
\hline & CGC AGT AGG CAC GAT GTT GTA \\
\hline \multirow{2}{*}{ iutA } & GGC TGG ACA TCA TGG GAA CTG G \\
\hline & CGT CGG GAA CGG GTA GAA TCG \\
\hline \multirow{2}{*}{$i b e A$} & AGG CAG GTG TGC GCC GCG TAC \\
\hline & TGG TGC TCC GGC AAA CCA TGC \\
\hline \multirow{2}{*}{$R f c$} & ATC CAT CAG GAG GGG ACT GGA \\
\hline & AAC CAT ACC AAC CAA TGC GAG \\
\hline \multirow{2}{*}{$\operatorname{traT}$} & GGT GTG GTG CGA TGA GCA CAG \\
\hline & CAC GGT TCA GCG ATC CCT GAG \\
\hline
\end{tabular}

of 38.9 years. Isolates were identified by MicroScan 4 (Dade Behring) automated system. The presence of $\beta$-lactamases and the minimum inhibitory concentration (MIC) were also determined for ampicillin, ampicillin/sulbactam, amoxicillin/clavulanic acid, aztreonam, imipenem, meropenem, piperacillin, piperacillin/tazobactam, ticarcillin/clavulanic acid, amikacin, gentamicin, tobramycin, ceftriaxone, ceftazidime, cefotaxime, cefoxitin, cephalotin, cefazolin, cefepime, cefuroxime, cefotetan, trimethoprim/sulfamethoxazole, ciprofloxacin, gatifloxacin, levofloxacin, and moxifloxacin. The antibiotic resistance was classified into sensitive, resistant, and ESBL (resistance due to $\beta$-lactamases). Pure cultures were maintained at $-70^{\circ} \mathrm{C}$ in brain-heart infusion 
TABLE 2: PCR conditions for each gene.

\begin{tabular}{|c|c|c|c|c|c|c|}
\hline Genes & $\begin{array}{c}\text { Inicial denaturation } \\
\left({ }^{\circ} \mathrm{C} / \mathrm{min}\right)\end{array}$ & $\begin{array}{c}\text { Denaturation } \\
\left({ }^{\circ} \mathrm{C} / \mathrm{s}\right)\end{array}$ & $\begin{array}{c}\text { Annealing } \\
\left({ }^{\circ} \mathrm{C} / \mathrm{s}\right)\end{array}$ & $\begin{array}{c}\text { Extension } \\
\left({ }^{\circ} \mathrm{C} / \mathrm{s}\right) \\
\end{array}$ & $\begin{array}{c}\text { Final extention } \\
\left({ }^{\circ} \mathrm{C} / \mathrm{min}\right)\end{array}$ & Cycles \\
\hline $\operatorname{ecp} A$ & $96 / 5$ & $94 / 30$ & $62 / 45$ & $72 / 45$ & $72 / 5$ & 35 \\
\hline $\operatorname{ecp} R-B$ & $95 / 5$ & $95 / 30$ & $57.5 / 33$ & $72 / 90$ & $75 / 5$ & 35 \\
\hline fimH & $96 / 5$ & $94 / 30$ & $65.5 / 30$ & $72 / 30$ & $72 / 5$ & 35 \\
\hline papC & $95 / 5$ & $94 / 30$ & $58.2 / 30$ & $72 / 40$ & $72 / 5$ & 40 \\
\hline$s f a S$ & $95 / 5$ & $94 / 30$ & $64 / 30$ & $72 / 25$ & $72 / 5$ & 35 \\
\hline $\operatorname{fog} G$ & $95 / 5$ & $95 / 30$ & $64 / 40$ & $72 / 30$ & $72 / 5$ & 30 \\
\hline$s f a / f o c D E$ & $95 / 5$ & $94 / 30$ & $65 / 30$ & $68 / 40$ & $72 / 3$ & 35 \\
\hline$c n f-1$ & $95 / 5$ & $94 / 30$ & $65.5 / 30$ & $72 / 30$ & $72 / 5$ & 35 \\
\hline hlyA & $95 / 5$ & $94 / 30$ & $63 / 30$ & $68 / 60$ & $72 / 5$ & 30 \\
\hline Multi1 ${ }^{*}$ & $95 / 5$ & $94 / 30$ & $67.1 / 30$ & $68 / 160$ & $72 / 5$ & 30 \\
\hline Multi2 $2^{* *}$ & $95 / 5$ & $94 / 30$ & $61.5 / 30$ & $68 / 180$ & $72 / 5$ & 35 \\
\hline$R f_{c}$ & $95 / 5$ & $95 / 30$ & $62.5 / 30$ & $72 / 60$ & $75 / 5$ & 40 \\
\hline
\end{tabular}

${ }^{*}$ Multiplex 1 for genes fyuA, iutA e ibeA. ${ }^{* *}$ Multiplex 2 for genes $c d t B, c v a C$ y traT.

broth/glycerol 50\%. The E. coli CFT073 uropathogenic strain was used as control strain.

2.2. Phylogenetic Groups and Virulence Factors. The PCR was performed with the GoTaq Flexi kit (Promega) according to the manufacturer's instructions. Phylogenetic groups were identified according to Clermont protocol [22]. 16 virulence genes of UPEC were included: fim $H, p a p C, s f a / f o c D E, s f a S$, focG, ecpA, ecpR-B, hlyA, cnf-1, cdt-B, cvaC, iutA, ibeA, $r f c$, trat $T$, and $f y u A$. PCR primers and conditions for each gene are described in Tables 1 and 2 [5, 17]. The ecp RB PCR was performed to overcome the possible variation of ecp $A$ which may give a false negative result of the $E$. coli common pilus [17]. All PCR products were visualized in agarose gel stained with ethidium bromide.

2.3. Statistical Analysis. To establish the results significance, the Fisher exact test was used. The level of significance was set at a $P$ value of $\leq 0.05$.

\section{Results}

The overall results of the isolates regarding the virulence genes, the phylogenetic group, and resistance profile are shown in Table 3 . Regarding the phylogenetic group, most of the isolates were (60) grouped into the B2 group (55.6\%), 33 isolates were classified as part of the A group (30.6\%), 10 isolates $(9.3 \%)$ to group B1, and 5 isolates $(4.6 \%)$ to group D.

3.1. Virulence Genes. Higher prevalence, above 50\%, was observed for the ecp, fim $H$, traT, sfa/focDE, and papC genes $(98.1 \%, 86.1 \%, 77.8 \%, 74.1 \%$, and $62 \%$, resp.). For $i u t A$ and fyuA genes prevalence was close to $50 \%$ ( $48.1 \%$ and $44.4 \%$, resp.), while the focG, sfaS, hlyA, cnf-1, cdt-B, cvaC, ibeA, and $r f c$ genesregistered prevalence lower than $10 \%(2.8 \%$, $1.9 \%, 7.4 \%, 6.5 \%, 0.9 \%, 2.8 \%, 2.8 \%$, and $0.9 \%$, resp.). Table 4 shows the distribution of virulence genes regarding the phylogenetic group. Most of the virulence factors associated with the phylogenetic group B2 were identified. The ecp ( $A$ and $R-B$ ) and fim $H$ genes are widely distributed among all groups (A 100\%/78.8\%, B1 100\%/70\%, B2 96.7\%/91.7\%, and D $100 \% / 100 \%$, resp.). The focG, sfaS, hlyA, cnf-1, $c d t-B$, and $c v a C$ genes were found only in isolates from the B2 group. The $r f_{c}$ gene was found in just one isolate from group B1. The hlyA, $c f t-1$, and traT genes were positively associated with group B2, and the iutA and $f y u A$ genes were negatively associated with group A.

3.2. Antibiotic Resistance. Above $50 \%$ of antibiotic resistance was observed for ampicillin/sulbactam (75.9\%), ampicillin (55.2\%), piperacillin (51.1\%), trimethoprim/sulfamethoxazole $(56.1 \%)$, ciprofloxacin $(62.3 \%)$, gatifloxacin $(62.5 \%)$, levofloxacin (60.2\%), and moxifloxacin (52.6\%). Sensitivity values above $50 \%$ were found to amoxicillin/clavulanic acid (68.8\%), aztreonam (78.4\%), imipenem (98.1\%), meropenem (100\%), piperacillin/tazobactam (86\%), ticarcillin/clavulanic acid (58.2\%), amikacin (93.5\%), gentamicin (72.2\%), tobramycin (56.5\%), ceftriaxone (78.1\%), ceftazidime (77.9\%), cefotaxime $(78.9 \%)$, cefoxitin (91.1\%), cefazolin (65.9\%), cefepime $(78.1 \%)$, cefuroxime $(71.1 \%)$, and cefotetan (98.4\%). Approximately $20 \%$ of isolates registered the presence of $\beta$-lactamases and around $20 \%$ were resistant to antimicrobials, as shown in Table 5. Isolates which displayed resistance to more than $\geq 3$ chemotherapeutic groups were considered multiresistant isolates, which represents $58 \%$. However no statistical relation was observed among multiresistance and phylogenetic group (Table 6).

\section{Discussion}

In this work $108 \mathrm{E}$. coli isolates were screened from female patients with an average age of 39 years; women were regarded as a productive population, for which urinary tract infections are considered a major cause of morbidity in our country and represent a huge economic impact $[23,24]$. 
TABLE 3: Virulence genes and phylogenetic group.

\begin{tabular}{|c|c|c|c|c|c|c|c|c|c|c|c|c|c|c|c|c|c|c|c|}
\hline \# Strain & $E c p$ & fimH & papC & $s f a / f o c D E$ & focG & $s f a S$ & hlyA & cnf-1 & $c d t B$ & $c v a C$ & iutA & $i b e A$ & $R f_{c}$ & $\operatorname{traT}$ & fyuA & chuA & $y j a$ & TSP & $\overline{P G}$ \\
\hline 1 & + & + & + & + & - & - & - & - & - & + & + & + & - & + & + & + & + & + & B2 \\
\hline 2 & + & + & - & - & - & - & - & - & - & - & + & - & - & + & + & + & - & - & $\mathrm{D}$ \\
\hline 3 & + & + & + & - & - & - & - & - & - & - & + & - & - & + & + & + & + & + & B2 \\
\hline 4 & + & + & + & + & - & - & - & - & - & - & - & - & - & - & - & - & + & - & A \\
\hline 5 & + & + & + & + & - & - & - & - & - & - & - & - & - & - & - & - & + & - & A \\
\hline 6 & + & + & + & - & - & - & - & - & - & - & + & - & - & + & + & - & + & - & A \\
\hline 7 & + & + & + & + & - & - & - & - & - & - & + & - & - & + & + & + & + & - & B2 \\
\hline 8 & + & - & + & + & - & - & - & - & - & - & + & - & - & + & + & + & + & + & B2 \\
\hline 9 & + & + & + & + & - & - & - & - & - & - & - & - & - & - & - & - & + & - & A \\
\hline 10 & + & + & + & + & - & - & - & - & - & - & - & - & - & + & + & + & + & - & B2 \\
\hline 11 & + & - & + & + & - & - & - & - & - & - & - & - & - & + & - & - & + & - & A \\
\hline 12 & - & + & + & - & - & - & - & - & - & - & + & - & - & + & + & + & + & + & B2 \\
\hline 13 & + & + & + & + & - & - & - & - & - & - & + & - & - & + & + & + & + & + & B2 \\
\hline 14 & + & + & + & + & - & - & - & - & - & - & - & - & - & - & - & + & + & - & B2 \\
\hline 15 & + & - & + & + & - & - & - & - & - & - & + & - & - & + & + & + & + & + & B2 \\
\hline 16 & + & + & + & + & - & - & - & - & - & - & + & - & - & + & - & - & + & - & A \\
\hline 17 & + & + & + & + & - & - & - & - & - & - & + & - & - & + & - & - & + & - & A \\
\hline 18 & + & + & + & + & - & - & - & - & - & - & + & - & - & + & + & + & + & - & B2 \\
\hline 19 & + & + & - & + & - & - & - & - & - & - & + & - & - & + & + & + & + & + & B2 \\
\hline 20 & + & + & + & + & - & - & - & - & - & - & - & - & - & + & - & - & + & + & B1 \\
\hline 21 & + & + & + & + & - & - & - & - & - & - & + & - & - & + & + & + & + & - & B2 \\
\hline 22 & + & + & + & + & - & - & - & - & - & - & - & - & - & + & - & + & + & + & B2 \\
\hline 23 & + & + & + & + & - & - & - & - & - & - & + & - & - & + & + & - & - & - & A \\
\hline 24 & + & + & + & + & - & - & - & - & - & - & - & - & - & + & - & + & + & + & B2 \\
\hline 25 & + & + & + & + & - & - & - & - & - & - & - & - & - & - & - & - & + & - & A \\
\hline 26 & - & + & + & + & - & - & - & - & - & - & - & - & - & + & - & + & + & - & B2 \\
\hline 27 & + & + & + & + & - & - & - & - & - & - & - & - & - & + & - & + & + & - & B2 \\
\hline 28 & + & + & - & + & - & - & - & - & - & - & + & - & - & + & + & + & + & + & B2 \\
\hline 29 & + & + & - & + & - & - & - & - & - & - & - & - & - & - & - & + & - & - & $\mathrm{D}$ \\
\hline 30 & + & + & + & + & + & - & + & + & - & - & - & - & - & + & + & + & + & + & B2 \\
\hline 31 & + & + & - & + & - & - & - & - & - & - & + & - & - & + & + & - & + & + & B1 \\
\hline 32 & + & + & + & + & - & - & + & + & - & - & - & - & - & + & - & + & + & + & B2 \\
\hline 33 & + & + & - & + & - & - & - & - & - & - & - & - & - & - & - & + & + & - & B2 \\
\hline 34 & + & + & + & - & - & - & - & - & - & - & - & - & - & + & + & - & + & - & A \\
\hline 35 & + & + & - & + & - & - & - & - & - & - & + & - & - & - & + & + & + & + & B2 \\
\hline 36 & + & + & - & + & - & - & - & - & - & - & - & - & - & + & - & + & - & - & $\mathrm{D}$ \\
\hline 37 & + & + & + & - & - & - & - & - & - & - & + & - & - & + & + & + & + & + & B2 \\
\hline 38 & + & + & + & - & - & - & - & - & - & - & - & - & - & + & - & + & + & + & B2 \\
\hline 39 & + & + & + & + & - & - & - & - & - & - & + & - & - & + & - & + & - & - & $\mathrm{D}$ \\
\hline 40 & + & + & + & + & - & - & - & - & - & - & - & - & - & + & - & + & + & - & B2 \\
\hline 41 & + & + & + & - & - & - & - & - & - & - & + & - & - & + & + & + & + & + & B2 \\
\hline 42 & + & + & - & - & - & - & - & - & - & - & + & - & - & + & + & + & + & + & B2 \\
\hline 43 & + & + & - & - & - & - & - & - & - & - & - & - & - & + & + & - & - & - & A \\
\hline 44 & + & + & - & - & - & - & - & - & - & - & - & - & - & - & - & - & + & - & A \\
\hline 45 & + & + & + & + & - & - & - & - & - & - & - & - & - & - & - & - & + & - & A \\
\hline 46 & + & + & + & + & - & - & - & - & - & - & + & - & - & + & + & + & + & - & B2 \\
\hline 47 & + & + & + & + & - & - & - & - & - & - & + & - & - & + & + & + & + & - & B2 \\
\hline 48 & + & + & + & + & - & - & - & - & - & - & - & - & - & - & - & - & + & - & A \\
\hline
\end{tabular}


TABle 3: Continued.

\begin{tabular}{|c|c|c|c|c|c|c|c|c|c|c|c|c|c|c|c|c|c|c|c|}
\hline \# Strain & $E c p$ & $\mathrm{fimH}$ & papC & $s f a / f o c D E$ & focG & $s f a S$ & hlyA & $c n f-1$ & $c d t B$ & cvaC & iutA & $i b e A$ & $R f_{c}$ & traT & fyuA & chuA & $y j a$ & TSP & $P G$ \\
\hline 49 & + & + & - & + & - & - & - & - & - & - & - & - & - & + & + & + & + & - & $\mathrm{B} 2$ \\
\hline 50 & + & + & - & + & - & - & - & - & - & - & - & - & - & + & + & - & + & - & A \\
\hline 51 & + & + & + & + & - & - & - & - & - & - & - & - & - & - & - & + & + & + & B2 \\
\hline 52 & + & + & + & + & - & - & - & - & - & - & - & - & - & - & - & - & + & - & A \\
\hline 53 & + & + & - & - & - & - & - & - & - & - & - & - & - & - & - & - & + & - & A \\
\hline 54 & + & + & + & - & - & - & - & - & - & - & - & - & - & + & + & + & + & - & $\mathrm{B} 2$ \\
\hline 55 & + & + & + & + & + & - & - & - & - & + & + & - & - & + & - & + & + & - & $\mathrm{B} 2$ \\
\hline 56 & + & + & + & + & - & - & - & - & - & - & + & - & - & + & - & - & + & - & A \\
\hline 57 & + & + & + & - & - & - & - & - & - & - & + & - & - & + & + & + & + & + & B2 \\
\hline 58 & + & - & + & - & - & - & - & - & - & - & - & - & - & - & - & - & - & + & $\mathrm{B} 1$ \\
\hline 59 & + & + & + & + & - & - & - & - & - & - & - & - & - & - & - & + & - & + & $\mathrm{D}$ \\
\hline 60 & + & - & + & - & - & - & - & - & - & - & + & - & - & - & - & - & + & - & A \\
\hline 61 & + & + & - & + & - & - & - & - & - & - & - & - & - & - & - & - & - & - & A \\
\hline 62 & + & + & - & - & - & - & - & - & - & - & + & - & - & + & + & + & + & + & B2 \\
\hline 63 & + & + & + & + & - & - & - & - & - & - & + & - & - & + & + & + & + & + & B2 \\
\hline 64 & + & + & - & - & - & - & - & - & - & - & + & - & - & + & + & + & + & + & B2 \\
\hline 65 & + & + & + & - & - & - & - & - & - & - & + & - & - & - & + & + & + & + & B2 \\
\hline 66 & + & + & + & + & + & - & + & + & - & - & - & - & - & + & - & + & + & + & B2 \\
\hline 67 & + & + & + & - & - & - & - & - & - & - & - & - & + & - & - & - & + & + & B1 \\
\hline 68 & + & + & - & + & - & - & - & - & - & - & - & - & - & + & - & + & + & + & B2 \\
\hline 69 & + & + & + & + & - & - & - & - & - & - & - & - & - & + & - & + & + & - & B2 \\
\hline 70 & + & + & - & - & - & - & - & - & - & - & - & - & - & + & + & - & + & - & A \\
\hline 71 & + & + & + & + & - & - & - & - & - & - & + & - & - & + & - & - & + & - & A \\
\hline 72 & + & + & - & + & - & - & - & - & - & - & - & - & - & - & - & - & - & - & A \\
\hline 73 & + & + & - & + & - & - & - & - & - & - & - & - & - & + & - & - & + & & A \\
\hline 74 & + & + & + & + & - & + & + & + & - & - & - & - & - & + & + & + & + & + & B2 \\
\hline 75 & + & + & - & - & - & - & - & - & - & - & + & - & - & + & + & + & + & - & B2 \\
\hline 76 & + & - & - & - & - & - & - & - & - & - & - & - & - & + & - & - & + & - & A \\
\hline 77 & + & + & - & + & - & - & - & - & - & - & - & - & - & + & + & + & + & - & B2 \\
\hline 78 & + & + & - & - & - & - & - & - & - & - & - & - & - & + & - & - & + & - & A \\
\hline 79 & + & + & - & + & - & - & - & - & - & - & + & - & - & - & - & + & + & - & B2 \\
\hline 80 & + & + & + & + & - & - & - & - & - & - & - & - & - & - & - & + & + & - & B2 \\
\hline 81 & + & + & + & + & - & - & - & - & - & - & + & - & - & + & + & + & + & + & B2 \\
\hline 82 & + & + & - & + & - & - & - & - & - & - & - & - & - & + & + & - & + & - & A \\
\hline 83 & + & + & + & + & - & + & + & + & - & - & + & - & - & + & + & + & + & + & B2 \\
\hline 84 & + & - & + & + & - & - & - & - & - & - & + & - & - & + & - & - & + & - & A \\
\hline 85 & + & - & + & + & - & - & - & - & - & - & - & - & - & + & - & - & + & - & A \\
\hline 86 & + & + & + & - & - & - & - & - & - & - & + & - & - & + & + & + & + & + & B2 \\
\hline 87 & + & + & - & - & - & - & - & - & - & - & + & - & - & + & - & - & + & - & A \\
\hline 88 & + & + & + & - & - & - & - & - & - & - & - & - & - & + & - & - & + & - & A \\
\hline 89 & + & + & - & + & - & - & - & - & - & - & - & - & - & + & + & - & + & + & B1 \\
\hline 90 & + & + & + & + & - & - & - & - & - & - & + & - & - & + & - & - & + & + & B1 \\
\hline 91 & + & - & - & + & - & - & - & - & - & - & - & - & - & - & - & - & + & + & B1 \\
\hline 92 & + & - & + & + & - & - & - & - & - & - & - & - & - & + & - & - & + & + & B1 \\
\hline 93 & + & + & + & + & - & - & - & - & - & - & - & - & - & + & - & - & + & + & B1 \\
\hline 94 & + & + & + & + & - & - & - & + & - & - & + & - & - & + & + & + & + & - & B2 \\
\hline 95 & + & + & + & + & - & - & - & - & - & - & + & - & - & + & + & + & + & + & B2 \\
\hline 96 & + & + & + & + & - & - & + & - & - & - & + & - & - & + & + & + & + & + & B2 \\
\hline 97 & + & - & - & + & - & - & - & - & - & - & - & - & - & + & - & - & + & - & A \\
\hline
\end{tabular}


TABLe 3: Continued.

\begin{tabular}{lccccccccccccccccccc}
\hline \# Strain & Ecp & fimH & papC & sfa/focDE & focG & sfaS & hlyA & cnf-1 & cdtB & cvaC & iutA & ibeA & Rfc & traT & fyuA & chuA & yja & TSP & PG \\
\hline 98 & + & + & + & + & - & - & + & + & + & - & + & - & - & + & + & + & + & + & $\mathrm{B} 2$ \\
99 & + & + & - & + & - & - & - & - & - & - & - & - & - & + & - & + & + & + & $\mathrm{B} 2$ \\
100 & + & + & - & + & - & - & - & - & - & - & + & - & - & + & + & + & + & + & $\mathrm{B} 2$ \\
101 & + & + & - & + & - & - & - & - & - & - & + & - & - & + & + & + & + & + & $\mathrm{B} 2$ \\
102 & + & + & - & - & - & - & - & - & - & - & + & + & - & + & - & - & + & + & $\mathrm{B} 1$ \\
103 & + & - & - & + & - & - & - & - & - & - & + & - & - & + & - & + & + & - & $\mathrm{B} 2$ \\
104 & + & - & - & + & - & - & - & - & - & + & + & + & - & + & - & + & + & + & $\mathrm{B} 2$ \\
105 & + & + & - & + & - & - & + & - & - & - & + & - & - & + & - & + & + & + & $\mathrm{B} 2$ \\
106 & + & + & - & + & - & - & - & - & - & - & + & - & - & + & - & + & + & + & $\mathrm{B} 2$ \\
107 & + & - & - & + & - & - & - & - & - & - & - & - & - & + & - & - & + & - & $\mathrm{A}$ \\
108 & + & - & - & + & - & - & - & - & - & - & - & - & - & + & - & + & + & + & $\mathrm{B} 2$ \\
\hline
\end{tabular}

Phylogenetic group (PG).

TABLE 4: Relation among phylogenetic group and virulence genes.

\begin{tabular}{|c|c|c|c|c|}
\hline \multirow[b]{2}{*}{ Gene } & \multicolumn{4}{|c|}{ Phylogenetic group $(n, \%)$} \\
\hline & $\begin{array}{c}\mathrm{A} \\
(n=33)\end{array}$ & $\begin{array}{c}\mathrm{B} 1 \\
(n=10)\end{array}$ & $\begin{array}{c}\mathrm{B} 2 \\
(n=60)\end{array}$ & $\begin{array}{c}\mathrm{D} \\
(n=5)\end{array}$ \\
\hline$E c p$ & $33(100)$ & $10(100)$ & $58(96.7)$ & $5(100)$ \\
\hline fimH & $\begin{array}{c}26 \\
(78.8)\end{array}$ & $7(70)$ & $55(91.7)$ & $5(100)$ \\
\hline papC & $19(57.6)$ & $6(60)$ & $40(66.7)$ & $2(40)$ \\
\hline$s f a / f o c D E$ & $\begin{array}{c}22 \\
(66.7)\end{array}$ & $7(60)$ & $47(78.3)$ & $4(80)$ \\
\hline focG & 0 & 0 & $3(5)$ & 0 \\
\hline$s f a S$ & 0 & 0 & $2(3.3)$ & 0 \\
\hline hlyA & 0 & 0 & $8(13.3)^{\mathrm{a}}$ & 0 \\
\hline$c n f-1$ & 0 & 0 & $7(11.7)^{\mathrm{a}}$ & 0 \\
\hline$c d t-B$ & 0 & 0 & $1(1.7)$ & 0 \\
\hline cvaC & 0 & 0 & $3(5)$ & 0 \\
\hline$i u t A$ & $\begin{array}{c}9 \\
(27.3)^{(a)}\end{array}$ & $3(30)$ & $38(63.3)^{\mathrm{a}}$ & $2(40)$ \\
\hline$i b e A$ & 0 & $1(10)$ & $2(3.3)$ & 0 \\
\hline$R f_{c}$ & 0 & $1(10)$ & 0 & 0 \\
\hline $\operatorname{traT}$ & $\begin{array}{c}21 \\
(63.6)\end{array}$ & $7(70)$ & $53(88.3)^{\mathrm{a}}$ & $3(60)$ \\
\hline fyuA & $\begin{array}{c}7 \\
(21.2)^{(a)}\end{array}$ & $2(20)$ & $38(63.3)^{\mathrm{a}}$ & $1(20)$ \\
\hline
\end{tabular}

${ }^{a} P$ values were calculated by comparison of each group with Fisher's exact test.

Statistic significance of $\leq 0.05^{(\mathrm{a})}$ negative association.

The predominant phylogenetic group was B2 (55.6\%), widely associated with pathogenic strains. In Spain and the United States similar results had been reported and also a lower percentage related to the phylogenetic group $\mathrm{D}$ $[6-8,18,25]$. The phylogenetic group $A$, associated with commensal strains, represents a $30.6 \%$, higher than in other studies, suggesting that the gastrointestinal tract is the main reservoir of strains that may be able to colonize the urinary tract in accordance to previous observations $[6,7,18,25]$. The B1 group (9.3\%) as a cause of urinary tract infections points out the high plasticity of the $E$ coli genome which allowed the presence of the fimH, papC, and ecp ( $A$ and $R B$ ) in percentages of $70 \%, 60 \%$, and $100 \%$, respectively. These genes are related to the ability to colonize the urinary tract epithelium [18, 22, 26-28].

Adhesins genes were present in high percentages: fimH (86.1\%), ecp (A y R-B) (98.1\%), and papC (62\%), this result could be related to the pathogenicity of the isolated strains as adherence is the most important pathogenicity determinant [4]. The fimH geneonce again was highly conserved in UTI isolates which confirms its crucial role during colonization of the urinary tract $[4,29-32]$. 
TABLE 5: Antibiotic resistance.

\begin{tabular}{|c|c|c|c|}
\hline Antibiotic (number of isolates) & $S(\%)$ & $R(\%)$ & ESBL (\%) \\
\hline ampicillin/sulbactam (108) & $26(24.1)$ & $82(75.9)$ & 0 \\
\hline ampicillin (97) & $22(22.7)$ & $54(55.7)$ & $21(21.9)$ \\
\hline amoxicillin/clavulinic acid (64) & $44(68.8)$ & $20(31.3)$ & 0 \\
\hline aztreonam (97) & $76(78.4)$ & 0 & $21(21.6)$ \\
\hline Imipenem (108) & $106(98.1)$ & $2(1.9)$ & 0 \\
\hline Meropenem (30) & $30(100)$ & 0 & 0 \\
\hline piperacillin/tazobactam (107) & $92(86)$ & $15(14)$ & 0 \\
\hline piperacillin (95) & $25(26.3)$ & $49(51.6)$ & $21(22.3)$ \\
\hline ticarcillin/clavulanic acid (91) & $53(58.2)$ & $38(41.8)$ & 0 \\
\hline amikacin (108) & $101(93.5)$ & $7(6.5)$ & 0 \\
\hline gentamicin (108) & $78(72.2)$ & $30(27.8)$ & 0 \\
\hline tobramycin (108) & $61(56.5)$ & $47(43.5)$ & 0 \\
\hline ceftriaxone (96) & $75(78.1)$ & 0 & $21(21.9)$ \\
\hline ceftazidime (95) & $74(77.9)$ & 0 & $21(22.1)$ \\
\hline cefotaxime $(76)$ & $60(78.9)$ & 0 & $16(21.1)$ \\
\hline cefoxitin (45) & $41(91.1)$ & $4(8.9)$ & 0 \\
\hline cephalotin (22) & $10(45.5)$ & $8(36.4)$ & $4(18.2)$ \\
\hline cefazolin (91) & $60(65.9)$ & $11(12.1)$ & $20(22)$ \\
\hline cefepime (96) & $75(78.1)$ & 0 & $21(21.9)$ \\
\hline cefuroxime (56) & $40(71.4)$ & $1(1.8)$ & $15(26.8)$ \\
\hline cefotetan (61) & $60(98.4)$ & $1(1.6)$ & 0 \\
\hline trimethoprim/sulfamethoxazole (107) & $47(43.9)$ & $60(56.1)$ & 0 \\
\hline ciprofloxacin (106) & $40(37.7)$ & $66(62.3)$ & 0 \\
\hline gatifloxacin (64) & $24(37.5)$ & $40(62.5)$ & 0 \\
\hline levofloxacin (108) & $43(39.8)$ & $65(60.2)$ & 0 \\
\hline moxifloxacin (19) & $9(47.4)$ & $10(52.6)$ & 0 \\
\hline
\end{tabular}

TABLE 6: Relation among multidrug resistance and phylogenetic group.

\begin{tabular}{lcc}
\hline Phylogenetic group & \multicolumn{1}{c}{ Multidrug resistance [positive isolates number (\%)] } \\
MDS $(n=63)$ & $22(34.9)$ \\
\hline A & No-MDS $(n=45)$ & $4(6.4)$ \\
B1 & $11(24.4)$ & $36(57.1)$ \\
B2 & $6(13.3)$ & $1(1.6)$ \\
D & $24(53.3)$ & $4(9)$ \\
\hline
\end{tabular}

MDS: multidrug sensitive. $P$ values were calculated by the Fisher's exact test for each group, none has statistical significance value $\leq 0.05$.

The ecp $(A$ and $R B)$ gene is associated with commensals and enteropathogenic strains; it was present in $98.1 \%$ of this study isolates and according to a similar observation in Portugal it was found in $100 \%$ of their isolates; it may be associated with UPEC $[17,22,24,28]$. The pap $C$ gene encodes an outer membrane protein essential for the fimbriae P biogenesis regulation. pap genes presence had been associated with pyelonephritis; therefore, higher percentages (over 50\%) suggest that the strains isolated from the Mexican population have greater capabilities to colonize kidneys and generate pyelonephritis [32, 33].

The $h l y A$ and $c n f-1$ genes showed a positive relationship with the $\mathrm{B} 2$ group; also they are associated with pathogenicity island PAI $\mathrm{II}_{\mathrm{T96}}$, and the iutA gene is associated with pathogenicity island PAI $\mathrm{I}_{\mathrm{CFT} 073}$ as well as with $h l y A$ and pap operon $[8,19,34-36]$.

The sfaS gene was found exclusively in hlyA and cnf1 positive isolates which could be linked to cystitits cases. This observation is in accordance with the previous report by Lloyd et al. [19].

The $c v a C$ gene was present in only three isolates traT positive. These genes are both located at the colV plasmid. traT is related to the phylogenetic group B2, and presumes an animal source. The iut $A$ and $f y u A$ genesalso showed a relation with the phylogenetic group B2 [37-39]. The ibeA gene, related to the B2 group, was found in an isolate identified as $\mathrm{B} 1$, a result that may start to change the previous assumption [40]. The $r f c$ gene was identified in just one isolate which 
indicates that the serogroup $\mathrm{O} 4$ was not the predominant serogroup in the population studied and that this result may need further serological confirmation $[5,41]$.

The treatment of choice for ITU is in order of importance: fluoroquinolones (ciprofloxacin), the trimethoprim/ sulfamethoxazole, cephalosporins, and penicillins (ampicillin) to which an increasingly developed resistance has been reported due mainly to the indiscriminated antibiotic use $[13,14]$. In this work it is confirmed the resistance previously reported values for trimethoprim/sulfamethoxazole (56.1\%) [14, 42]; for ciprofloxacin (62.3\%), gatifloxacin, levofloxacin, and moxifloxacin, resistance was always above $50 \%$. Positive isolates to $h l y A, c n f-1$, and/or papC genes were susceptible to fluoroquinolones, results similar to those of Piatti et al. [43]. Besides in Mexico, the previously reported E. coli resistance profile included ampicillin, piperacillin, fluoroquinolones, and trimethoprim/sulfamethoxazole which are considered to be first-line choices $[13,44,45]$. Additionally, serotype 025bST131 has been reported to be within the Mexican population which has been associated with plasmid mediated quinolone resistance [46]. We had identified multidrug resistance of the E. coli strains causing UTI in $58 \%$ of the isolates which belonged mainly to group B2, result which kept our attention.

\section{Conclusion}

This work confirms that most of the isolates associated with urinary tract infections belong to the phylogenetic group B2 and in a lesser extent to group D. Also they displayed a great number of virulence genes. However, commensal strains may also be the cause of UTI. According to our results most parts of the isolates have the ability to colonize the kidneys as they have a high incidence of the $p a p C$ gene. The $h l y A$ and cnf-1 genes encoding toxins and fyuA iutA and siderophores encoding genes are tightly associated with the phylogenetic group B2.

E. coli has successfully adapted to host's conditions and to the general medical practices as we may observe the high resistance to trimethoprim/sulfamethoxazole and fluoroquinolones, especially on the most frequently isolated phylogenetic groups.

Finally, these results reinforce international knowledge on antimicrobial resistance and the high rate of multidrug resistance found invites us to encourage population awareness of the proper use of antimicrobials.

\section{Conflict of Interests}

The authors declare that there is no conflict of interests regarding the publication of this paper.

\section{Acknowledgment}

This work was supported by the Consejo Nacional de Ciencia y Tecnología, Grant CONACyT-87586-Salud 2008.

\section{References}

[1] J. B. Kaper, J. P. Nataro, and H. L. T. Mobley, "Pathogenic Escherichia coli," Nature Reviews Microbiology, vol. 2, no. 2, pp. 123-140, 2004.

[2] G. Croxall, J. Hale, V. Weston et al., "Molecular epidemiology of extraintestinal pathogenic Escherichia coli isolates from a regional cohort of elderly patients highlights the prevalence of ST131 strains with increased antimicrobial resistance in both community and hospital care settings," Journal of Antimicrobial Chemotherapy, vol. 66, no. 11, pp. 2501-2508, 2011.

[3] O. Clermont, S. Bonacorsi, and E. Bingen, "Rapid and simple determination of the Escherichia coli phylogenetic group," Applied and Environmental Microbiology, vol. 66, no. 10, pp. 4555-4558, 2000.

[4] M. A. Mulvey, "Adhesion and entry of uropathogenic Escherichia coli," Cellular Microbiology, vol. 4, no. 5, pp. 257-271, 2002.

[5] J. R. Johnson and A. L. Stell, "Extended virulence genotypes of Escherichia coli strains from patients with urosepsis in relation to phylogeny and host compromise," The Journal of Infectious Diseases, vol. 181, no. 1, pp. 261-272, 2000.

[6] E. Moreno, J. R. Johnson, T. Pérez, G. Prats, M. A. Kuskowski, and A. Andreu, "Structure and urovirulence characteristics of the fecal Escherichia coli population among healthy women," Microbes and Infection, vol. 11, no. 2, pp. 274-280, 2009.

[7] E. Moreno, A. Andreu, C. Pigrau, M. A. Kuskowski, J. R. Johnson, and G. Prats, "Relationship between Escherichia coli strains causing acute cystitis in women and the fecal E. coli population of the host," Journal of Clinical Microbiology, vol. 46, no. 8, pp. 2529-2534, 2008.

[8] D. W. Hilbert, T. E. Paulish, E. Mordechai, M. E. Adelson, and J. P. Trama, "O serogroups, phylogeny, and virulence factors of cervicovaginal and rectal Escherichia coli isolates," European Journal of Clinical Microbiology and Infectious Diseases, vol. 27, no. 12, pp. 1265-1268, 2008.

[9] N. Ahmed, U. Dobrindt, J. Hacker, and S. E. Hasnain, “Genomic fluidity and pathogenic bacteria: applications in diagnostics, epidemiology and intervention," Nature Reviews Microbiology, vol. 6, no. 5, pp. 387-394, 2008.

[10] M. Mellata, K. Ameiss, H. Mo, and R. Curtiss III, "Characterization of the contribution to virulence of three large plasmids of avian pathogenic Escherichia coli $\chi 7122$ (O78:K80:H9)," Infection and Immunity, vol. 78, no. 4, pp. 1528-1541, 2010.

[11] M. N. Chulain, A.-M. Murray, G. Corbett-Feeney, and M. Cormican, "Antimicrobial resistance in E. Coli associated with urinary tract infection in the west of Ireland," Irish Journal of Medical Science, vol. 174, no. 4, pp. 6-9, 2005.

[12] J. R. Johnson, M. A. Kuskowski, A. Gajewski, D. F. Sahm, and J. A. Karlowsky, "Virulence characteristics and phylogenetic background of multidrug-resistant and antimicrobialsusceptible clinical isolates of Escherichia coli from across the United States, 2000-2001," The Journal of Infectious Diseases, vol. 190, no. 10, pp. 1739-1744, 2004.

[13] J. Molina-López, G. Aparicio-Ozores, R. M. Ribas-Aparicio et al., "Drug resistance, serotypes, and phylogenetic groups among uropathogenic Escherichia coli including O25-ST131 in Mexico City, Journal of Infection in Developing Countries, vol. 5, no. 12, pp. 840-849, 2011. 
[14] A. Moura, A. Nicolau, T. Hooton, and J. Azeredo, "Antibiotherapy and pathogenesis of uncomplicated UTI: difficult relationships," Journal of Applied Microbiology, vol. 106, no. 6, pp. 17791791, 2009.

[15] D. de Backer, T. Christiaens, S. Heytens, A. de sutter, E. E. Stobberingh, and G. Verschraegen, "Evolution of bacterial susceptibility pattern of Escherichia coli in uncomplicated urinary tract infections in a country with high antibiotic consumption: a comparison of two surveys with a 10 year interval," Journal of Antimicrobial Chemotherapy, vol. 62, no. 2, pp. 364-368, 2008.

[16] B. K. Hong, H. P. Chi, J. K. Chung, E.-C. Kim, G. A. Jacoby, and D. C. Hooper, "Prevalence of plasmid-mediated quinolone resistance determinants over a 9-year period," Antimicrobial Agents and Chemotherapy, vol. 53, no. 2, pp. 639-645, 2009.

[17] D. Blackburn, A. Husband, Z. Saldaña et al., "Distribution of the Escherichia coli common pilus among diverse strains of human enterotoxigenic E. coli," Journal of Clinical Microbiology, vol. 47, no. 6, pp. 1781-1784, 2009.

[18] E. Moreno, A. Andreu, T. Pérez, M. Sabaté, J. R. Johnson, and G. Prats, "Relationship between Escherichia coli strains causing urinary tract infection in women and the dominant faecal flora of the same hosts," Epidemiology and Infection, vol. 134, no. 5, pp. 1015-1023, 2006.

[19] A. L. Lloyd, D. A. Rasko, and H. L. T. Mobley, "Defining genomic islands and uropathogen-specific genes in uropathogenic Escherichia coli," Journal of Bacteriology, vol. 189, no. 9, pp. 35323546, 2007.

[20] R. Raz, Y. Gennesin, J. Wasser et al., "Recurrent urinary tract infections in postmenopausal women," Clinical Infectious Diseases, vol. 30, no. 1, pp. 152-156, 2000.

[21] R. Fronzes, H. Remaut, and G. Waksman, "Architectures and biogenesis of non-flagellar protein appendages in Gramnegative bacteria," The EMBO Journal, vol. 27, no. 17, pp. 22712280, 2008.

[22] M. A. Rendón, Z. Saldaña, A. L. Erdem et al., "Commensal and pathogenic Escherichia coli use a common pilus adherence factor for epithelial cell colonization," Proceedings of the National Academy of Sciences of the United States of America, vol. 104, no. 25, pp. 10637-10642, 2007.

[23] SNdV, "Panorama epidemiológico de las infecciones de vías urinarias en México 2003-2008," Epidemiológica, vol. 26, no. 52, 2009.

[24] Geografía INEGI, "Censo de población y vivienda 2010 panorarma sociodemográfico de México," 2011.

[25] A. Narciso, F. Nunes, T. Amores, L. Lito, J. Melo-Cristino, and A. Duarte, "Persistence of uropathogenic Escherichia coli strains in the host for long periods of time: relationship between phylogenetic groups and virulence factors," European Journal of Clinical Microbiology and Infectious Diseases, vol. 31, no. 6, pp. 1211-1217, 2012.

[26] K. Ejrnaes, "Bacterial characteristics of importance for recurrent urinary tract infections caused by Escherichia coli," Danish Medical Bulletin, vol. 58, no. 4, Article ID B4187, 2011.

[27] J. D. Sobel, "Pathogenesis of urinary tract infection. Role of host defenses," Infectious Disease Clinics of North America, vol. 11, no. 3, pp. 531-549, 1997.

[28] J. Xicohtencatl-Cortes, V. Monteiro-Neto, M. A. Ledesma et al., "Intestinal adherence associated with type IV pili of enterohemorrhagic Escherichia coli O157:H7," The Journal of Clinical Investigation, vol. 117, no. 11, pp. 3519-3529, 2007.

[29] D. M. Guyer, N. W. Gunther IV, and H. L. T. Mobley, "Secreted proteins and other features specific to uropathogenic
Escherichia coli," The Journal of Infectious Diseases, vol. 183, supplement 1, pp. S32-S35, 2001.

[30] L. Emody, M. Kerényi, and G. Nagy, "Virulence factors of uropathogenic Escherichia coli," International Journal of Antimicrobial Agents, vol. 22, supplement 2, pp. S29-S33, 2003.

[31] J. M. Bower, D. S. Eto, and M. A. Mulvey, "Covert operations of uropathogenic Escherichia coli within the urinary Tract," Traffic, vol. 6, no. 1, pp. 18-31, 2005.

[32] E.-M. Antao, L. H. Wieler, and C. Ewers, "Adhesive threads of extraintestinal pathogenic Escherichia coli," Gut Pathogens, vol. 1, article 22, 2009.

[33] M. C. Lane and H. L. T. Mobley, "Role of P-fimbrial-mediated adherence in pyelonephritis and persistence of uropathogenic Escherichia coli (UPEC) in the mammalian kidney," Kidney International, vol. 72, no. 1, pp. 19-25, 2007.

[34] S. Yamamoto, "Molecular epidemiology of uropathogenic Escherichia coli," Journal of Infection and Chemotherapy, vol. 13, no. 2, pp. 68-73, 2007.

[35] M. Bingen-Bidois, O. Clermont, S. Bonacorsi et al., "Phylogenetic analysis and prevalence of urosepsis strains of Escherichia coli bearing pathogenicity island-like domains," Infection and Immunity, vol. 70, no. 6, pp. 3216-3226, 2002.

[36] S. M. Soto, S. Zúñiga, P. Ulleryd, and J. Vila, "Acquisition of a pathogenicity island in an Escherichia coli clinical isolate causing febrile urinary tract infection," European Journal of Clinical Microbiology and Infectious Diseases, vol. 30, no. 12, pp. 1543-1550, 2011.

[37] V. Hancock, L. Ferrières, and P. Klemm, "The ferric yersiniabactin uptake receptor FyuA is required for efficient biofilm formation by urinary tract infectious Escherichia coli in human urine," Microbiology, vol. 154, no. 1, pp. 167-175, 2008.

[38] T. E. Clarke, L. W. Tari, and H. J. Vogel, "Structural biology of bacterial iron uptake systems," Current Topics in Medicinal Chemistry, vol. 1, no. 1, pp. 7-30, 2001.

[39] M. C. Rowe, H. L. Withers, and S. Swift, "Uropathogenic Escherichia coli forms biofilm aggregates under iron restriction that disperse upon the supply of iron," FEMS Microbiology Letters, vol. 307, no. 1, pp. 102-109, 2010.

[40] D. M. Gordon, O. Clermont, H. Tolley, and E. Denamur, "Assigning Escherichia coli strains to phylogenetic groups: multi-locus sequence typing versus the PCR triplex method," Environmental Microbiology, vol. 10, no. 10, pp. 2484-2496, 2008.

[41] S. Lukomski, R. A. Hull, and S. I. Hull, "Identification of the $\mathrm{O}$ antigen polymerase $(\mathrm{rfc})$ gene in Escherichia coli $\mathrm{O} 4$ by insertional mutagenesis using a nonpolar chloramphenicol resistance cassette," Journal of Bacteriology, vol. 178, no. 1, pp. 240-247, 1996.

[42] M. T. Blahna, C. A. Zalewski, J. Reuer, G. Kahlmeter, B. Foxman, and C. F. Marrs, "The role of horizontal gene transfer in the spread of trimethoprim-sulfamethoxazole resistance among uropathogenic Escherichia coli in Europe and Canada," Journal of Antimicrobial Chemotherapy, vol. 57, no. 4, pp. 666-672, 2006.

[43] G. Piatti, A. Mannini, M. Balistreri, and A. M. Schito, "Virulence factors in urinary Escherichia coli strains: phylogenetic background and quinolone and fluoroquinolone resistance," Journal of Clinical Microbiology, vol. 46, no. 2, pp. 480-487, 2008.

[44] J. L. Arredondo-García and C. F. Amábile-Cuevas, "High resistance prevalence towards ampicillin, co-trimoxazole and ciprofloxacin, among uropathogenic Escherichia coli isolates in Mexico City," Journal of Infection in Developing Countries, vol. 2, no. 5, pp. 350-353, 2008. 
[45] J. L. Arredondo-García, D. Soriano-Becerril, F. SolórzanoSantos, A. Arbo-Sosa, R. Coria-Jiménez, and P. Arzate-Barbosa, "Resistance of uropathogenic bacteria to first-line antibiotics in mexico city: a multicenter susceptibility analysis," Current Therapeutic Research-Clinical and Experimental, vol. 68, no. 2, pp. 120-126, 2007.

[46] F. Reyna-Flores, H. Barrios, U. Garza-Ramos et al., "Molecular epidemiology of Escherichia coli O25b-ST131 isolates causing community-acquired UTIs in Mexico," Diagnostic Microbiology \& Infectious Disease, vol. 76, no. 3, pp. 396-398, 2013. 


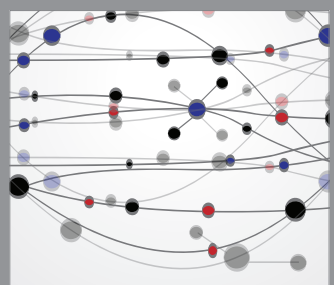

The Scientific World Journal
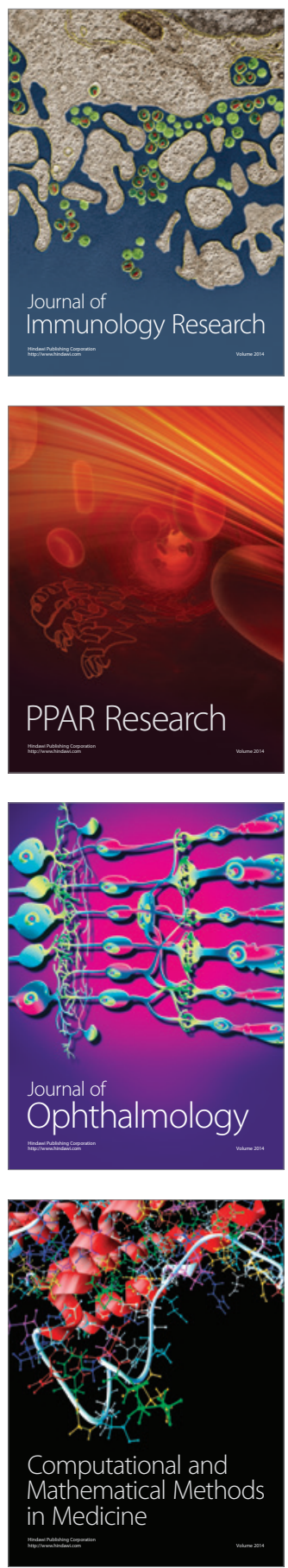

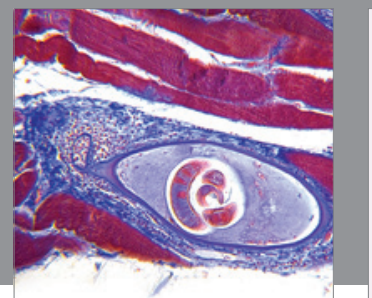

Gastroenterology

Research and Practice
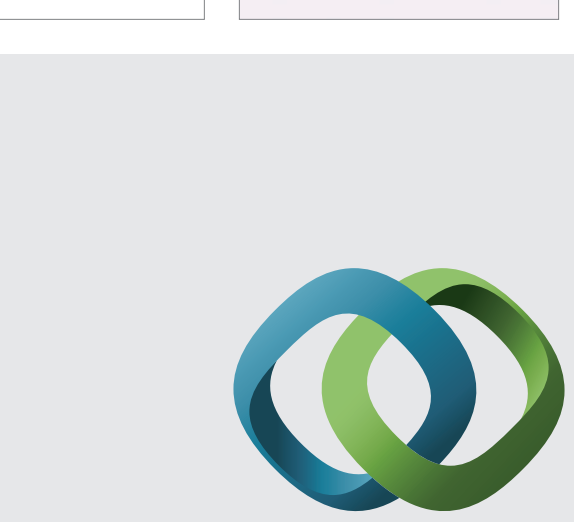

\section{Hindawi}

Submit your manuscripts at

http://www.hindawi.com
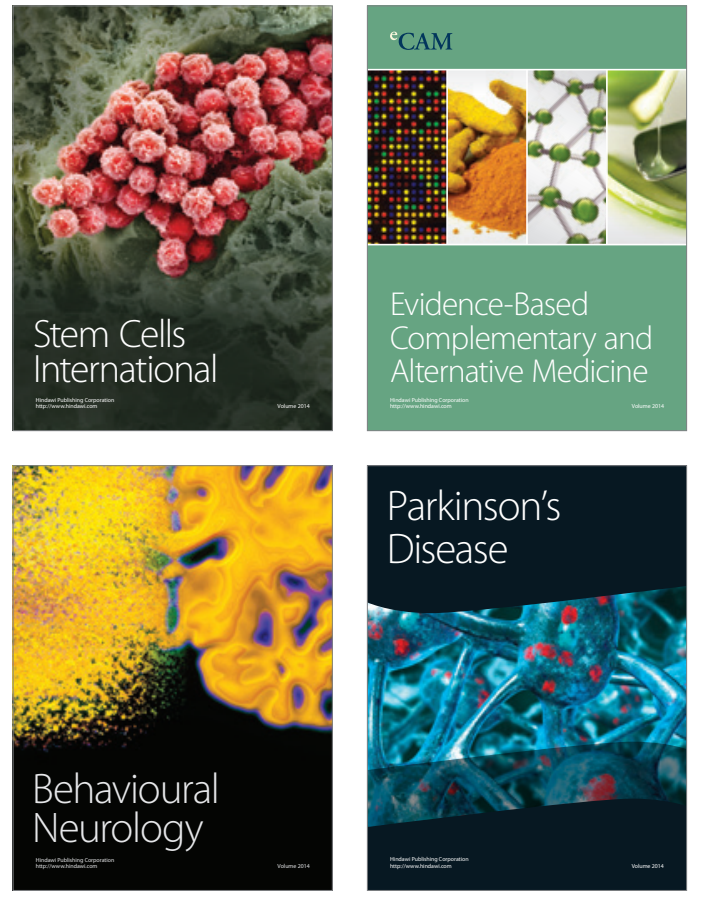
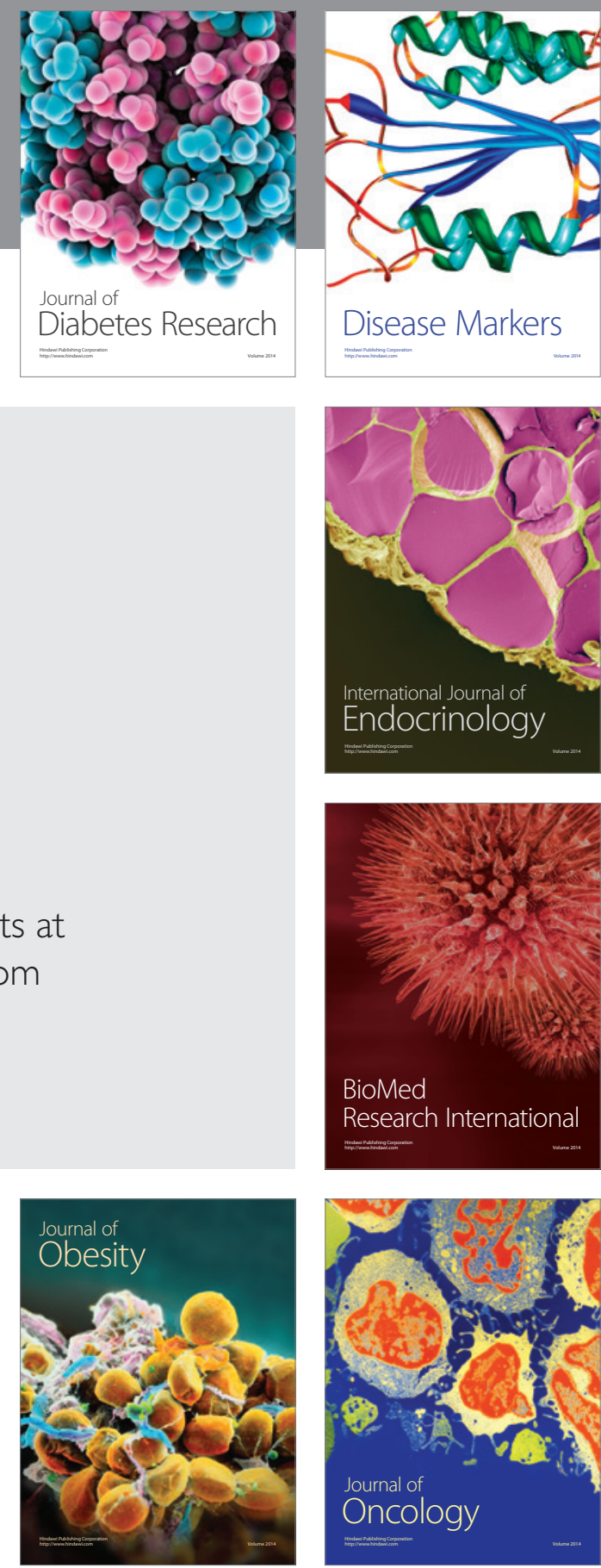

Disease Markers
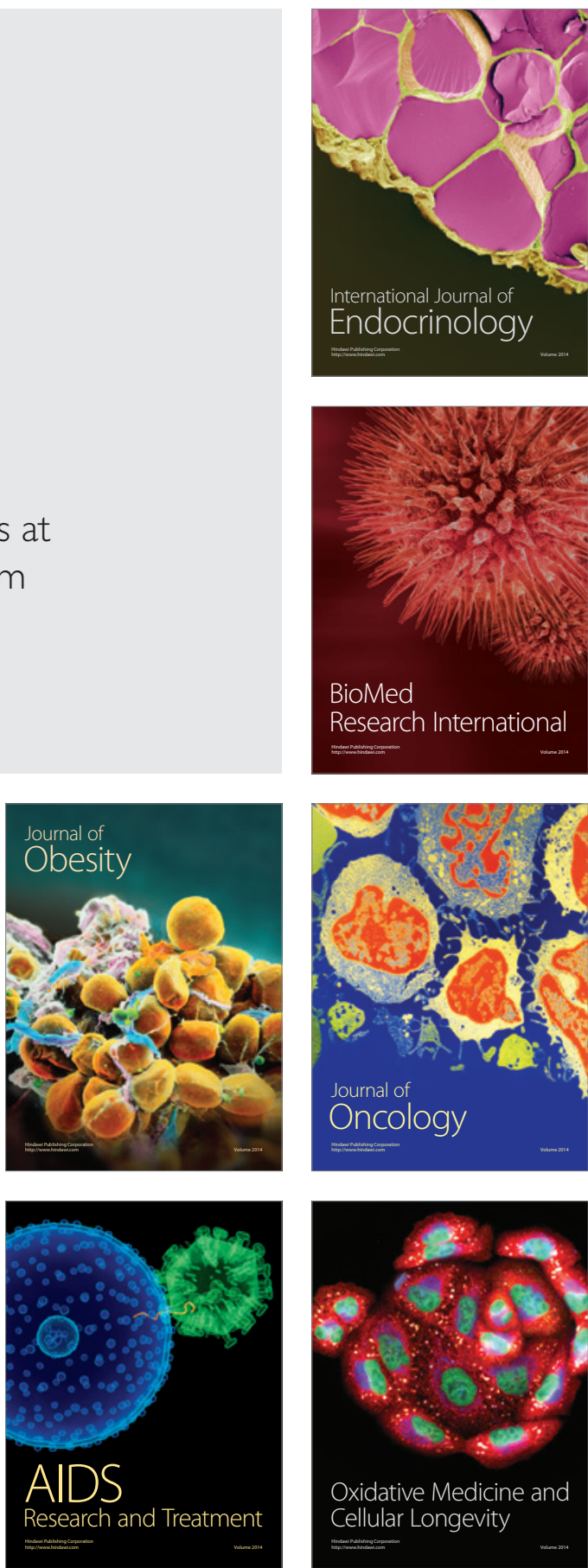